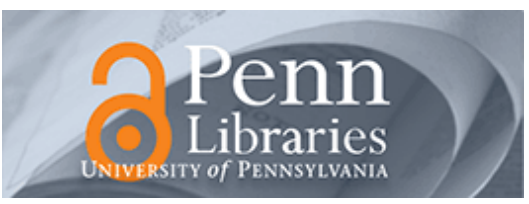

University of Pennsylvania

ScholarlyCommons

September 2003

\title{
A silicon implementation of the thalamic low threshold calcium current
}

Kai M. Hynna

University of Pennsylvania

Kwabena A. Boahen

University of Pennsylvania, boahen@seas.upenn.edu

Follow this and additional works at: https://repository.upenn.edu/be_papers

\section{Recommended Citation}

Hynna, K. M., \& Boahen, K. A. (2003). A silicon implementation of the thalamic low threshold calcium current. Retrieved from https://repository.upenn.edu/be_papers/19

Copyright 2003 IEEE. Reprinted from Proceedings of the 25th Annual International Conference of the IEEE Engineering in Medicine and Biology Society 2003, pages 2228-2231.

Publisher URL: http://ieeexplore.ieee.org/xpl/tocresult.jsp?isNumber=28615\&page=4

This material is posted here with permission of the IEEE. Such permission of the IEEE does not in any way imply IEEE endorsement of any of the University of Pennsylvania's products or services. Internal or personal use of this material is permitted. However, permission to reprint/republish this material for advertising or promotional purposes or for creating new collective works for resale or redistribution must be obtained from the IEEE by writing to pubs-permissions@ieee.org. By choosing to view this document, you agree to all provisions of the copyright laws protecting it.

This paper is posted at ScholarlyCommons. https://repository.upenn.edu/be_papers/19

For more information, please contact repository@pobox.upenn.edu. 


\title{
A silicon implementation of the thalamic low threshold calcium current
}

\author{
Abstract \\ A silicon model of the thalamic low threshold calcium current is presented. The channel current (IT) is the \\ product of an activation and inactivation current, normalized by their sum. The individual currents are \\ modeled by a simple current-mirror integrator circuit. A modified differential pair controls the threshold of \\ activation while a leak transistor added to the inactivation mirror controls the rate of inactivation and \\ deinactivation. The dynamics of IT are the result of the interaction between the fast activation and slow \\ inactivation currents. By adjusting the base level of the activation current, we can realize a \\ hyperpolarization activated cation current $(\mathrm{Ih})$, responsible for rhythmic bursting in thalamic cells. By \\ attaching the circuit to a constant leak integrate-and-fire neuron, we demonstrate in silicon both burst and \\ tonic firing modes.

\section{Keywords} \\ neuromorphic, silicon, $T$ current, thalamus

\section{Comments} \\ Copyright 2003 IEEE. Reprinted from Proceedings of the 25th Annual International Conference of the IEEE \\ Engineering in Medicine and Biology Society 2003, pages 2228-2231. \\ Publisher URL: http://ieeexplore.ieee.org/xpl/tocresult.jsp?isNumber=28615\&page=4 \\ This material is posted here with permission of the IEEE. Such permission of the IEEE does not in any way \\ imply IEEE endorsement of any of the University of Pennsylvania's products or services. Internal or \\ personal use of this material is permitted. However, permission to reprint/republish this material for \\ advertising or promotional purposes or for creating new collective works for resale or redistribution must \\ be obtained from the IEEE by writing to pubs-permissions@ieee.org. By choosing to view this document, \\ you agree to all provisions of the copyright laws protecting it.
}




\title{
A Silicon Implementation of the Thalamic Low Threshold Calcium Current
}

\author{
K. M. Hynna ${ }^{1}$, K. A. Boahen ${ }^{1}$ \\ 'Department of Bioengineering, University of Pennsylvania, Philadelphia, PA, USA
}

\begin{abstract}
A silicon model of the thalamic low threshold calcium current is presented. The channel current $\left(I_{T}\right)$ is the product of an activation and inactivation current, normalized by their sum. The individual currents are modeled by a simple current-mirror integrator circuit. A modified differential pair controls the threshold of activation while a leak transistor added to the inactivation mirror controls the rate of inactivation and deinactivation. The dynamics of $I_{T}$ are the result of the interaction between the fast activation and slow inactivation currents. By adjusting the base level of the activation current, we can realize a hyperpolarization activated cation current $\left(I_{h}\right)$, responsible for rhythmic bursting in thalamic cells. By attaching the circuit to a constant leak integrate-and-fire neuron, we demonstrate in silicon both burst and tonic firing modes.
\end{abstract}

Keywords-neuromorphic, silicon, $\mathbf{T}$ current, thalamus

\section{INTRODUCTION}

Almost all sensory input to the brain passes through the thalamus on the way to the cortex. Often referred to as the "gateway" to the cortex, it is believed by some to be simply a relay point for sensory information. However, recent studies suggest that the thalamus, with the massive feedback it receives from the cortex, may play an active role in information processing in the awake state [2-4].

One of the distinguishing features of the neurons within the thalamus is their ability to elicit a high-frequency burst of action potentials. The burst is generated by the activation of a low threshold $\mathrm{Ca}^{++}$channel called a $\mathrm{T}$ channel. The current passed by this channel, $I_{T}$, is activated at subthreshold levels and inactivates, when the cell. is depolarized, on a time scale of tens of milliseconds. Deinactivation of the channel requires the cell to remain at hyperpolarized membrane potentials for an extended period of time. Full deinactivation typically requires hundreds of milliseconds [5].

Given the voltage dependence of $\mathrm{I}_{\mathrm{T}}$, the resting membrane potential can have a dramatic effect on the firing mode of the relay neuron. Cortical afferents activate metabotropic glutamate receptors in the relay cell which decrease a membrane $\mathrm{K}^{+}$leak conductance. This decrease in the cell leak slowly depolarizes the cell resulting in the inactivation of $I_{T}$ before it can properly activate. As a result, the cells firing mode switches from burst to tonic, where the cell responds like a plain integrate-and-fire neuron $[6,7]$.

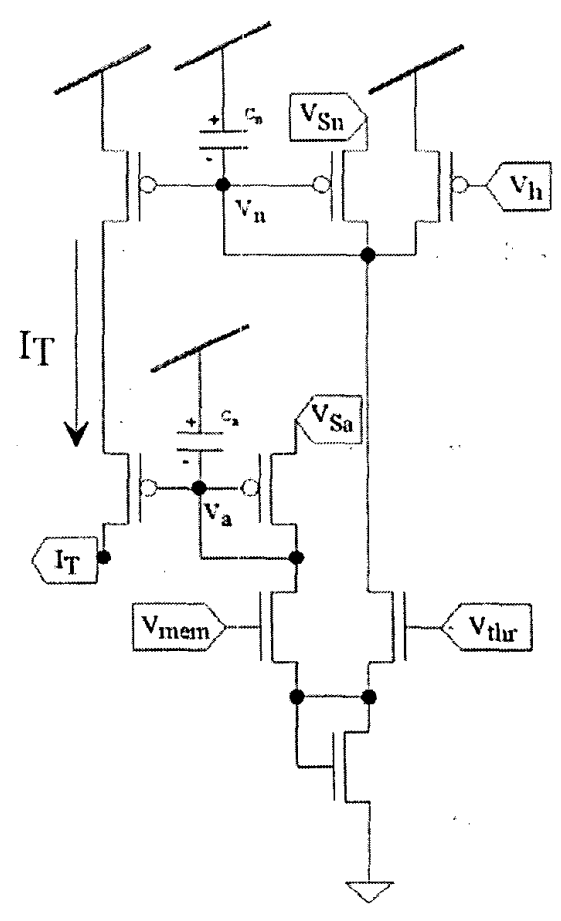

Fig. 1. Circuit Schematic of T Channel

Another current in thalamic cells commonly coupled with $I_{T}$ is the hyperpolarization-activated cation current $\left(I_{h}\right)$. This current activates at hyperpolarized membrane levels, depolarizing the cell. Coupled with $\mathrm{I}_{\mathrm{T}}$, this current can lead to rhythmic bursting within the relay neuron[8].

Here we demonstrate a simple silicon model of $\mathrm{I}_{\mathrm{T}}$. Recordings from a fabricated silicon neuron model are presented to demonstrate the bursting capabilities as a result of the addition of this channel.

\section{METHODOLOGY}

Fig. 1 shows the schematic for our $\mathrm{T}$ current. Computational models generally use the product of an activation and inactivation variable to represent their current. Our model uses the product of an activation and inactivation current normalized to their sum, computed by connecting these transistors in series[9]. The temporal dynamics of both of these currents are realized using a 




Fig. 2. Low threshold calcium burst in the silicon neuron. The cell starts in a hyperpolarized state and bursts in response to a depolarizing input.

current mirror integrators. As its name implies, the current mirror outputs a current equal in magnitude but opposite in sign to the input current. A capacitor on the gate of the input diode provides an integration time constant while a source tilt on this transistor $\left(V_{S a}\right.$ and $V_{S n}$ in fig. 1) provides a constant gain to the output. The addition of a constant leak $\left(V_{h}\right)$ to the inactivation mirror controls the rates of inactivation and deinactivation in the channel.

Current into the activation and inactivation integrators (the latter we will call the deinactivation current) is controlled by the three transistors at the bottom of fig. 1. The ratio of the output currents from this circuit, called a modified differential pair, is controlled exponentially by the difference in the two gates voltages $\left(\mathrm{V}_{\text {mem }}\right.$ and $\left.\mathrm{V}_{\mathrm{thr}}\right) . \mathrm{V}_{\mathrm{thr}}$ is fixed and thus provides a set voltage threshold the membrane must cross for channel activation. The behavior of the $T$ current is controlled by the fast dynamics of the activation mirror and the slower dynamics of the inactivation mirror.

At hyperpolarized levels (i.e. $V_{\text {mem }}<V_{\text {thr }}$ ), the current into the activation side of the modified differential pair is negligible to that on the inactivation side: the channel begins to deinactivate. The rate of deinactivation depends on the difference between the deinactivation current and the leak current (i.e., the level of hyperpolarization). The amount of deinactivation depends both on the length of time the cell is hyperpolarized and the level of hyperpolarization.

As the cell depolarizes, the activation current begins to increase and the deinactivation current decreases. Once the deinactivation current is reduced below the leak in the inactivation mirror, the channel begins to inactivate. If the cell depolarizes too slowly, then the channel will have inactivated before it even had a chance to activate. However, if the cell is depolarized sufficiently fast, then the activation current will turn on before the inactivation mirror

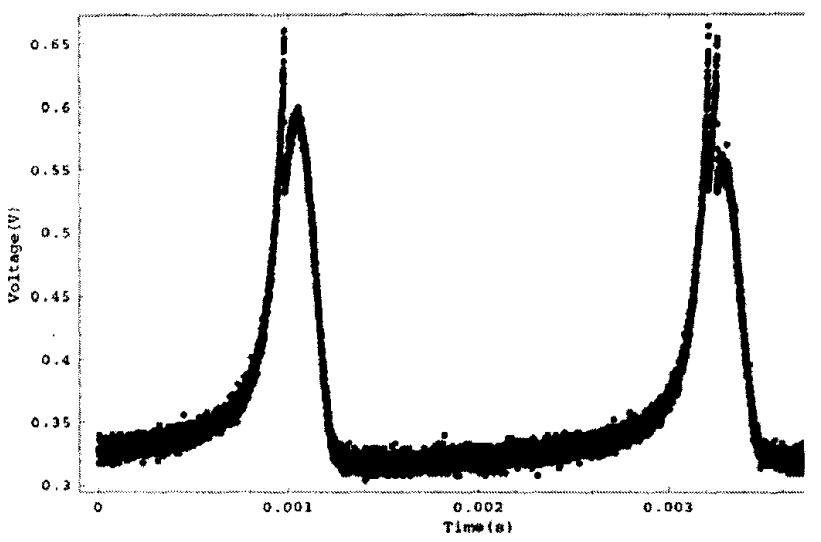

Fig. 3. Rhythmic bursting. At the end of a burst, $I_{\mathrm{T}}$ is inactivated and the cell hyperpolarizes. As $I_{T}$ deinactivates, $I_{h}$ increases. Once the neuron leak current is surpassed, the cell begins to depolarize and the cycle repeats.

is allowed to discharge and a $\mathrm{T}$ current is produced, a similar trait is seen in real relay cells [10].

Another interesting feature of this circuit is its ability to emulate a hyperpolarization-induced cation current $\left(\mathrm{I}_{h}\right)$ as well. The source tilt on the input diode of the activation mirror $\left(V_{\mathrm{Sa}}\right.$ in fig. 1) provides not only a gain on the input current, but a base level of activation. Typically, in an inactivated state, $I_{T}$ is limited by the output of the inactivation mirror due to cell depolarization. When the cell is hyperpolarized, the inactivation current increases due to deinactivation, but $I_{T}$ still remains off now due to the lack of activation. However, by increasing the base activation level, we can introduce a current once $I_{T}$ has deinactivated, regardless of the hyperpolarized state of the cell. This causes the cell to start to depolarize and then eventually burst, a behaviour very similar to that induced by $I_{h}$.

On chip, we attached this channel to a simple neuron model. The neuron is a constant-leak integrate-and-fire neuron: once the input current surpasses the leak, the neuron fires at a rate proportional to the level of the input current. To record from the neuron, we instrumented the voltages from the membrane capacitor and the activation and inactivation mirrors. The chip was fabricated in TSMC's $0.25 \mu \mathrm{m}$ process through MOSIS.

\section{RESULTS}

Fig. 2 shows the membrane voltage of our integrateand-fire neuron during a burst. The cell begins in a hyperpolarized state, having been there long enough to deinactivate $\mathrm{I}_{\mathrm{T}}$. As the membrane voltage rises, due to a change in the input, the positive feedback from the activation of $\mathrm{I}_{\mathrm{T}}$ eventually kicks in initiating the burst. The cell stops bursting once $\mathrm{I}_{\mathrm{T}}$ sufficiently inactivates to drop below the cell leakage, at which point the membrane voltage 


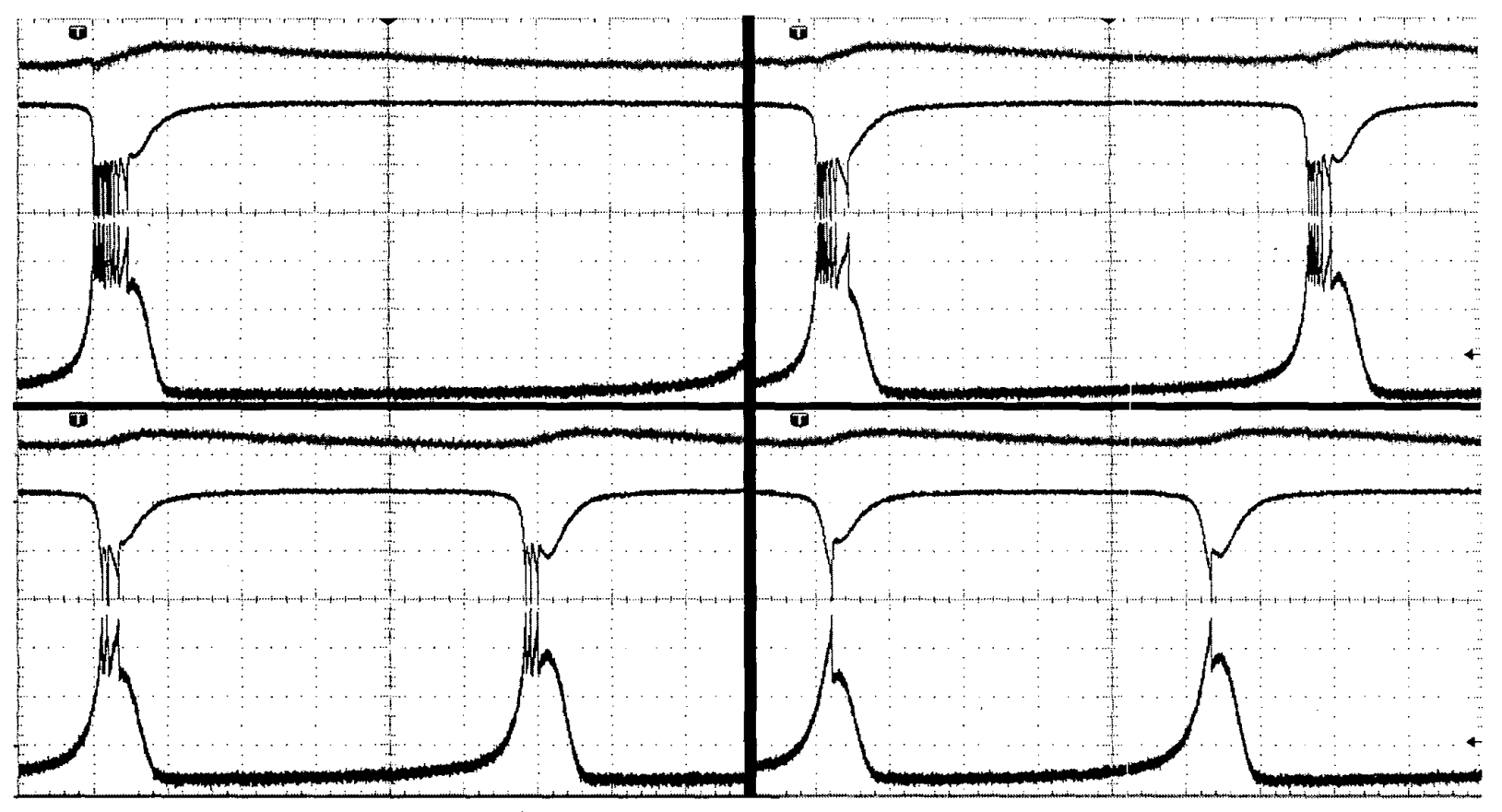

Fig. 4. Rhythmic bursting for four different leakage currents (decreasing from left to right, top to bottom). In each quadrant, the traces from top to bottom are the inactivation, activation and membrane voltages. As the leakage decreases, the inter-burst interval also decreases due to the fact that less deinactivation is required for $\mathrm{I}_{h}$ to overcome the total leak current (compare the top trace in each quadrant). This results in a weaker $\mathrm{I}_{\mathrm{T}}$ current, and as a result the number of spikes per burst decreases $(11,6,3,1)$. Horizontal resolution is $400 \mu \mathrm{s} /$ division. The membrane voltage has a vertical resolution of

$100 \mathrm{mV} /$ division at an offset of $390 \mathrm{mV}$. The activation and inactivation voltages have vertical resolutions of $50 \mathrm{mV} / \mathrm{division}$ and $20 \mathrm{mV} / \mathrm{division}$ respectively. Both are at an offset of $770 \mathrm{mV}$. Leakage current was decreased by about $1.5 \%$ between top-left and bottom-right traces.

hyperpolarizes. The number of $\mathrm{Na}^{+}$spikes in the burst is controlled by two factors: 1) the time constant of inactivation and 2) the initial level of deinactivation. The former depends on the inactivation leak $V_{h}$. The latter, as mentioned previously, depends on both on the level and duration of hyperpolarization. If the channel has not deinactivated fully, then less time is needed to inactivate it.

Fig. 3 demonstrates the rhythmic bursting ability of our neuron. Raising the base activation level (by increasing the source tilt $\mathrm{V}_{\mathrm{Sa}}$ ) eventually produces a hyperpolarization activated current $\left(\mathrm{I}_{\mathrm{h}}\right)$ that is dependent on the deinactivation of $\mathrm{I}_{\mathrm{T}}$. Immediately after a burst, the cell hyperpolarizes due to $I_{T}$ inactivation. As the $T$ channel deinactivates, the output series transistors starts to pass more and more current due to the increased base activation level. Eventually, $I_{h}$ surpasses the leak and the cell slowly begins to depolarize. Once the membrane voltage approaches the activation threshold, $\mathrm{I}_{\mathrm{T}}$ is activated and produces another burst and the cycle begins again.

In the rhythmic bursting case, because our $I_{h}$ current is now coupled with the deinactivation of $I_{T}$, the number of spikes is dependent on the difference between the input current and the membrane leak. $\mathrm{I}_{h}$ only needs to be large enough to surpass this difference, after which the membrane voltage will begin to rise stopping the deinactivation. Fig. 4 shows the rhythmic bursting of the cell in response to increasing leakage current. The top left figure, with the smallest input, has the largest interburst interval and the largest number of spikes. As we increase the input current the interburst interval reduces as do the number of spikes. We move from 11 spikes per burst to roughly one spike. Comparing deinactivation levels we see the level of deinactivation increases with greater leak, and as a result so do the number of spikes.

Fig. 5 shows the response frequency of the cell to varying current step sizes. To switch between firing modes, the leak of the neuron was reduced until the cell depolarized sufficiently to inactivate $\mathrm{I}_{\mathrm{T}}$. In tonic (single-spike) mode, after surpassing the membrane leak $(\sim 0.54 \mathrm{nA})$, the neuron responds linearly in frequency to the input current. During the burst mode, a larger current was needed to elicit a burst $(>0.66 \mathrm{nA})$, due to the larger leak in the neuron. Once the threshold is passed the neuron responds with a highfrequency burst.

\section{DISCUSSION AND CONCLUSION}

We have presented a silicon model of the low threshold calcium current found in thalamic cells. We demonstrate that the cell has many burst properties similar to their biological counterparts. In addition, the design contained an 



Fig. 5. Frequency vs. Input plots for the neuron in its two firing modes. The frequency is calculated from the first interspike interval in response to a step current. In the single-spike mode (left), the neuron response is linear in frequency with respect to the input current. The threshold for activity is the point at which the input surpasses the leak. In burst mode, the threshold is much higher due to the presence of a stronger leak. However, once activated, the cell responds with a high-frequency burst due to the activation of $\mathrm{I}_{\mathrm{T}}$. Compare with Fig. $2 \mathrm{e}$ in [1].

intrinsic $\mathrm{I}_{\mathrm{h}}$ current that allowed for rhythmic bursting of the cell. Hooked up to a simple neuron model, the cell was able to rhythmically burst on its own.

One of the major deficiencies with this design is reliance of the dynamics of inactivation and deinactivation on the difference between two currents. One problem encountered frequently encountered in analog VLSI design is that of transistor mismatch. The fabrication of silicon chips introduces variations in many of the physical parameters both between chips and within a chip. This means that two circuits, though identical in their layout, may respond quite differently. Identically sized and biased transistors may vary by as much as $30 \%$ in current when operated in the subthreshold region. Since this design relies on the small difference between two currents to achieve long time constants, a population of these cells would respond quite differently to the same input. Thus, some cells would burst as desired, other would burst with faster time constants, others may activate a weak $\mathrm{Ca}^{++}$spike with no $\mathrm{Na}^{+}$spikes, others may do nothing at all.

Fig. 5 shows another difficulty faced by our subthreshold designs: our ability to achieve the slower time constants seen in biology. Our firing rates were approximately three orders of magnitude higher than those recorded in the guinea-pig thalamus (compare fig. 5 to fig. $2 \mathrm{E}$ in [1]). On-chip capacitance is a function of area; unfortunately, so is the cost of fabrication. Using smaller currents is a possible solution, but newer technologies have larger intrinsic leakage currents associated with a transistor, setting a lower bound on how small a current we can generate.

Future designs will take these issues into account. Our goal is eventually to build an artificial thalamic network, with both relay and reticular cells. This would provide us with the opportunity to study network properties in a population of cells, both in the forward transmission of retinal information and the feedback effects of the cortical inputs.

\section{REFERENCES}

[1] D. A. McCormick and H. R. Feeser, "Functional implications of burst firing and single spike activity in lateral geniculate relay neurons," Neuroscience, vol. 39, pp. 103-13, 1990.

[2] P. C. Murphy and A. M. Sillito, "Corticofugal feedback influences the generation of length tuning in the visual pathway," Nature, vol. 329, pp. 727-9, 1987.

[3] A. M. Sillito, H. E. Jones, G. L. Gerstein, and D. C. West, "Feature-linked synchronization of thalamic relay cell firing induced by feedback from the visual cortex," Nature, vol. 369, pp. 479-82, 1994

[4] J. Cudeiro and A. M. Sillito, "Spatial frequency tuning of orientation-discontinuity-sensitive corticofugal feedback to the cat lateral geniculate nucleus," J Physiol (Lond), vol. 490, pp. 481-92, 1996.

[5] J. R. Huguenard and D. A. McCormick, "Simulation of the currents involved in rhythmic oscillations in thalamic relay neurons," J Neurophysiol, vol. 68, pp. 1373-83, 1992.

[6] D. W. Godwin, J. W. Vaughan, and S. M. Sherman, "Metabotropic glutamate receptors switch visual response mode of lateral geniculate nucleus cells from burst to tonic," $J$ Neurophysiol, vol. 76, pp. 1800-16, 1996.

[7] D. A. McCormick and M. von Krosigk, "Corticothalamic activation modulates thalamic firing through glutamate "metabotropic" receptors," Proc Natl Acad Sci US A, vol. 89, pp. 2774-8, 1992.

[8] D. A. McCormick and H. C. Pape, "Properties of a hyperpolarization-activated cation current and its role in rhythmic oscillation in thalamic relay neurones," J Physiol, vol. 431, pp. 291-318, 1990.

[9] T. Delbruck, "'Bump' circuits for computing similarity and dissimilarity of analog voltages," presented at Neural Networks, 1991., IJCNN-91-Seattle International Joint Conference on, 1991.

[10] X. J. Zhan, C. L. Cox, J. Rinzel, and S. M. Sherman, "Current clamp and modeling studies of low-threshold calcium spikes in cells of the cat's lateral geniculate nucleus," $J$ Neurophysiol, vol. 81, pp. 2360-73, 1999. 